\title{
FDG-PET/CT assessment of misty mesentery: Feasibility for distinguishing viable mesenteric malignancy from stable conditions.
}

\section{$\operatorname{AUTHOR}(S)$ :}

Nakatani, Koya; Nakamoto, Yuji; Togashi, Kaori

\section{CITATION:}

Nakatani, Koya .... [et al]. FDG-PET/CT assessment of misty mesentery: Feasibility for distinguishing viable mesenteric malignancy from stable conditions.. European journal of radiology 2013, 82(8): e380-e385

\section{ISSUE DATE:}

2013-08

URL:

http://hdl.handle.net/2433/176336

\section{RIGHT:}

(c) 2013 Elsevier Ireland Ltd.; この論文は出版社版でありません。引用の 際には出版社版をご確認ご利用ください。; This is not the published version. Please cite only the published version. 
FDG-PET/CT assessment of misty mesentery: feasibility for distinguishing viable mesenteric malignancy from stable conditions

Koya Nakatani, Yuji Nakamoto, Kaori Togashi

Department of Diagnostic Imaging and Nuclear Medicine, Kyoto University Graduate School of Medicine

Koya Nakatani: Corresponding author

Department of Diagnostic Imaging and Nuclear Medicine, Kyoto University Hospital, 54 Shogoin-kawahara-cho, Sakyo-Ku, Kyoto, 606-8507 Japan Phone: +81-75-751-3762 Fax: +81-75-771-9709 e-mail: koyakn@kuhp.kyoto-u.ac.jp Yuji Nakamoto:

54 Shogoin-kawahara-cho, Sakyo-Ku, Kyoto, 606-8507 Japan

Phone: +81-75-751-3762 Fax: +81-75-771-9709 e-mail: ynakamo1 @kuhp.kyoto-u.ac.jp Kaori Togashi:

54 Shogoin-kawahara-cho, Sakyo-Ku, Kyoto, 606-8507 Japan

Phone: +81-75-751-3760 Fax: +81-75-771-9709 e-mail: ktogashi@kuhp.kyoto-u.ac.jp 


\section{ABSTRACT}

Purpose: "Misty mesentery" is a CT appearance of mesenteric fat changes with increased attenuation often existing with swelled mesenteric lymph nodes. We evaluated diagnostic performance of FDG-PET/CT in distinguishing viable malignant disorders from benign conditions in misty mesentery.

Materials and Methods: 4236 FDG-PET/CT images were reviewed to identify patients with appearances of misty mesentery. Only the initial examinations were evaluated. Patients undergoing chemotherapy and/or radiotherapy within 3 months, patients with bulky mesenteric mass, and patients without follow-up examinations were excluded. Maximum short-axis diameter of mesenteric nodules (Diam-max) and maximum standardized uptake value (SUVmax) for mesenteric abnormalities were measured, and the diagnostic performance to differentiate between viable malignancy and stable lesions was assessed by receiver-operating characteristic (ROC) analysis, based on final diagnoses obtained by histology or follow-up examinations. Their significance was assessed by multivariate logistic regression.

Results: 71 studies met the inclusion criteria with confirmed diagnoses (13 viable malignancies; 58 stable lesions). Of the 13 malignant cases, 12 were lymphoma. ROC curves indicated an optimal Diam-max cut-off value of > $10 \mathrm{~mm}$ and SUVmax cut-off of $\geq 3.0$, with area under the curve of 0.961 and 0.926 , respectively. Using the optimal Diam-max cut-off, sensitivity and specificity were $69 \%$ and $98 \%$, respectively. Using 
the optimal SUVmax cut-off, they were $85 \%$ and $98 \%$, respectively. The combination of either Diam-max $>10 \mathrm{~mm}$ or SUVmax $\geq 3.0$ had a sensitivity of $92 \%$. Both Diammax and SUVmax were significant independent factors for predicting malignancy.

Conclusions: FDG-PET/CT is feasible for identifying viable malignancy in misty mesentery.

Key words:

FDG, PET/CT, mesentery, mesenteric panniculitis, lymphoma 


\section{Introduction}

A mesentery, suspending small intestine, consists of a fusion of two layers of peritoneum, between which there exist mesenteric vessels, lymphatic vessels, nerves, and variable amounts of fat [1]. 'Misty mesentery' is the term introduced by Mindelzun et al. describing a computed tomography (CT) appearance of mesenteric fat changes with increased attenuation when fluid, fibrosis, or inflammatory or neoplastic cell infiltration spreads between two layers of mesentery [2]. It often exists along with mesenteric lymph node enlargement and mesenteric vessel dilatation of various degrees (Fig. 1). One of the major causes is represented by mesenteric panniculitis. Mesenteric panniculitis, the synonyms for which include sclerosing mesenteritis, mesenteric lipodystrophy, liposclerotic mesenteritis, and retractile panniculitis, is histologically characterized by diffuse, localized, or multi-nodular thickening of mesentery with an infiltration of inflammatory cells such as myofibroblasts and foamy macrophages [3]. The primary causes of misty mesentery include various conditions besides panniculitis: lymphedema, edema, hemorrhage, trauma, and neoplasm [2]. The most common tumor involving mesentery is non-Hodgkin lymphoma, and it is an important differential diagnosis for patients with misty mesentery. CT features of misty mesentery are usually considered to be nonspecific among these conditions. Moreover, it often presents as an incidental finding in asymptomatic patients. Though some authors stated 'fat ring' sign might be an important CT finding for diagnosing mesenteric panniculitis, this finding 
seems not to be entirely specific [4]. Corwin et al. stated mesenteric lymph nodes larger than a short-axis diameter of $10 \mathrm{~mm}$ in misty mesentery were specific for malignancy [5]. However, their sensitivity seems not to be sufficient.

Positron emission tomography (PET) / CT using fluorine-18 fluorodeoxyglucose (FDG) has widely been used in clinical oncology. Zissin et al. studied 33 FDG-PET/CT scans of 19 oncologic patients with findings of mesenteric panniculitis. They suggested mesenteric FDG uptake would also be useful for the detection of co-existing mesenteric tumor involvement [6]. Since FDG-PET/CT provides both morphological and metabolic information, a better impact might be expected in the management of misty mesentery. Therefore, the purpose of our study was to analyze the diagnostic performance of FDGPET/CT, using a combination of anatomical and metabolic information, in distinguishing viable malignant disorders from benign conditions in those with misty mesentery that is not confined to oncologic patients.

\section{Patients and Methods}

\section{Study inclusion criteria and exclusion criteria}

This retrospective study was approved by the ethics committee in our institute and written informed consent was obtained from all participants for their data access. Between May 2009 and March 2011, a total of 4236 whole-body FDG-PET/CT examinations of 3399 patients were examined in our single institute. The analysis 
population of this study was patients with a CT appearance of misty mesentery on their PET/CT images among all these eligible examinations. The identification of misty mesentery was determined by experienced radiologists according to the following criteria: normal mesenteric fat appears as a homogeneous area of low attenuation (typically -100 to -160 Hounsfield units (HU)), which is about the same as the attenuation of subcutaneous and retroperitoneal fat, whereas misty mesentery was defined as an area of adipose tissue density around mesenteric vessels which had a visually perceptible area of increased attenuation (typically -40 to $-60 \mathrm{HU}$ ) relative to the attenuation of subcutaneous and retroperitoneal fat, as well as a generally segmental distribution of changes along the branches of the mesenteric vessels with or without interspersed soft tissue density nodules in the mesentery [1, 2, 5, 7]. CT appearance of misty mesentery according to these criteria was identified in 209 exams. Of these, 153 exams held as the initial examinations were included in the study.

The following exams were excluded: (a) exams for patients with a past history of receiving any chemotherapy or radiotherapy within 3 months $(n=40)$; (b) exams for patients with discrete bulky masses larger than a short-axis diameter of $2 \mathrm{~cm}$ in the mesentery $(n=7)$; (c) exams for patients who never underwent any follow-up abdominal examinations (including CT and PET/CT) or histological confirmation for mesentery $(n=33)$. Accordingly, 73 PET/CT exams with an appearance of misty mesentery were found to be eligible (Fig. 2). 


\section{PET/CT scanning protocol}

Before FDG administration, all patients were asked to fast for at least 4 hours. Administered FDG activity for each patient was $3.7 \mathrm{MBq} / \mathrm{kg}$ body weight. PET/CT images were acquired 1 hour after the administration. All studies were performed using a combined PET/CT scanner (Discovery ST Elite; GE Healthcare Ltd., Amersham Place, UK) integrating a bismuth germinate crystal PET system with a 16-slice multi-detectorrow $\mathrm{CT}$ scanner. CT data acquisition for attenuation correction and for precise anatomical information was performed under the following protocol: $20-100 \mathrm{~mA}$ tube current (using Auto-mA setting with Noise Index of 30), $120 \mathrm{kV}$ tube voltage, $0.6 \mathrm{sec}$ tube rotation, 1.75 helical pitch, and $3.75 \mathrm{~mm}$ section thickness. Images were then reconstructed by filtered back projection into $512 \times 512$ pixel images. PET emission scanning was performed sequentially after the CT scans with an acquisition time of 2-3 min per bed position, and then the images were reconstructed using a three-dimensional iterative reconstruction algorithm called VUE Point Plus (GE Healthcare Ltd., Amersham Place, UK).

\section{PET/CT image interpretation and data analyses}

The largest short-axis diameter of all mesenteric lymph nodes or soft tissue nodules (Diam-max) in misty mesentery was recorded for each analysis population. Preceding research suggested it was representative anatomical information correlated with the risk of malignancy [5]. These short-axis diameters were measured in the transaxial planes of 
the CT images as part of FDG-PET/CT. The highest maximum standardized uptake value (SUVmax) of mesenteric nodes or soft tissues in misty mesentery was also recorded as the representative metabolic information. In measuring SUVmax of the nodules, PET/CT fusion images were used to discriminate them from physiological FDG uptake including intestinal uptake. Both parameters were measured by using Centricity PACS and Centricity AW suite 2.0 workstations (GE Healthcare Ltd., Amersham Place, UK).

\section{Standard of reference}

Based on subsequent CT or PET/CT images at more than 6 months follow-up or histological confirmation, misty mesentery was judged as 'viable malignancy' or 'stable lesions', according to the following criteria: in the mesentery with viable malignancy, either mesenteric abnormalities' natural enlargement on the images within the follow-up period or their reduction synchronized with favorable response to chemotherapy against known coexistent malignancy should be observed in at least one mesenteric nodule. Otherwise, direct histological confirmation about the mesenteric abnormalities was required. On the other hand, when all of the mesenteric abnormalities were unchanged or had spontaneously decreased (without any therapeutic intervention) on the images throughout the follow-up period, they were regarded as stable lesions. If there were cases which were difficult to be judged into either category, they were regarded as 'inconclusive results' and were excluded from the following statistical analyses. 


\section{Statistical analyses}

The average of Diam-max in the cases with 'viable malignancy' and the average in the cases with 'stable lesions' were compared by using two-tailed Mann-Whitney U-test. The averages of SUVmax in the two groups were also compared by using the same statistics. Receiver-operating characteristic (ROC) analyses were performed to determine optimal cut-off values of Diam-max and SUVmax that would best discriminate between viable malignancy and stable lesions in each model. Using each cut-off value, the sensitivity, specificity, and accuracy for detecting viable malignancy were calculated, respectively. The differences between these results obtained from each cut-off value were assessed with the McNemar test. In addition, the sensitivity, specificity, and accuracy, using the combination of the optimal cut-off values of Diammax and SUVmax, were calculated. Pearson's correlation coefficient was checked to evaluate the correlation between Diam-max and SUVmax, and their significance as an independent predictor was assessed by multivariate logistic regression using backward stepwise methods. Statistical significance was defined as $P$ less than 0.05 . Prism 5 (GraphPad Software, La Jolla, CA, USA) was used for all statistical analyses except logistic regression. IBM SPSS Statistics 20 (IBM Inc., Armonk, NY, USA) was used for logistic regression. 
Final diagnoses as the reference standard were confirmed in 71 patients among the 73 eligible individuals; the other two patients were considered to have 'inconclusive results' (one of them was because of discrepant response to chemotherapy against ovarian cancer among her mesenteric nodules of almost the same size, and the other was because of spontaneous regression after transient enlargement of his mesenteric nodules), and therefore they were excluded from the statistical analyses. The profiles of the 71 patients analyzed are summarized in Table 1.

Among the 71 patients, 'viable malignancy' in mesentery was confirmed in 13 patients. Two of them were diagnosed by directed biopsy of a mesenteric nodule in which malignant lymphoma was histologically confirmed. Of these patients, another 10 were diagnosed as lymphoma by biopsy of cervical node, axillary node, inguinal node, or gastric wall (Fig. 3): as for these 10 patients, mesenteric tumor reduction synchronized with favorable response to chemotherapy was confirmed in 8 patients, and mesenteric tumor enlargement on follow-up images was identified in two patients. The other one of the 13 patients with 'viable malignancy' had local recurrence of pancreatic cancer at the root of mesentery: her misty mesentery was diminished at follow-up examination after salvage chemoradiation for the recurrence. Meanwhile, 12 of the 13 patients with viable malignancy in the mesentery had malignant lymphoma as a consequence. The histology included follicular lymphoma $(n=8)$, diffuse large B-cell lymphoma $(n=3)$, and angioimmunoblastic T-cell lymphoma $(n=1)$. Moreover, 7 of 
the 12 patients had new cases of lymphoma that had not been diagnosed before the present PET/CT exams.

Conversely, 'stable lesions' were confirmed in 58 patients. Of these, 56 patients were confirmed by remaining unchanged (one of whom also had a benign biopsy result (Fig. 4)) and the remaining two patients were confirmed by spontaneous tumor regression.

As shown in Fig. 5, misty mesentery with viable malignancy had a tendency to have larger mesenteric nodule with higher FDG uptake. The average of Diam-max in the cases with 'viable malignancy' $(11.8 \pm 3.0 \mathrm{~mm}$ : mean \pm standard deviation $)$ was significantly higher than the average in the cases with 'stable lesions' $(4.6 \pm 2.2 \mathrm{~mm})(\mathrm{P}$ $<0.0001)$. The average of SUVmax in 'viable malignancy' $(6.6 \pm 5.2)$ was significantly higher than the average in 'stable lesions' $(1.6 \pm 0.6)(\mathrm{P}<0.0001)$. ROC curves indicated an optimal Diam-max cut-off value of $>10 \mathrm{~mm}$ and an optimal SUVmax cutoff value of $\geq 3.0$ to differentiate between 'viable malignancy' and 'stable lesions'. Area under the ROC curve was calculated as 0.961 [95\% confidence interval $=0.91,1.01]$ for Diam-max and $0.926[0.83,1.03]$ for SUVmax (Fig. 6). Using the optimal Diam-max cutoff value, the sensitivity, specificity, and accuracy were $69 \%, 98 \%$, and $93 \%$, respectively. Using the optimal SUVmax cut-off value, they were $85 \%, 98 \%$, and $96 \%$, respectively (Table 2). The optimal SUVmax as a cut-off value had slightly higher sensitivity without decreasing specificity, but the differences did not reach statistical significance according to the McNemar test. 
There was moderate $(r=0.69)$ but significant $(\mathrm{p}<0.0001)$ correlation between Diammax and SUVmax of the mesenteric nodules (Fig. 5). Multivariate logistic regression revealed both Diam-max and SUVmax were significant independent factors for predicting the presence of viable malignancy (Table 3). When the combination of both a Diam-max cut-off value of $>10 \mathrm{~mm}$ and a SUVmax cut-off value of $\geq 3.0$ was used as positive criteria, the sensitivity, specificity, and accuracy were $62 \%, 100 \%$, and $93 \%$. If either the Diam-max cut-off value of $>10 \mathrm{~mm}$ or SUVmax cut-off value of $\geq 3.0$ was to be sufficient for a positive result, they were 92\%, 97\%, and 96\%, respectively (Table 2). When compared with the diagnostic performance of the optimal Diam-max cutoff value respectively, the differences did not reach statistical significance according to the McNemar test, either.

\section{Discussion}

The combination of morphological and metabolic information from FDG-PET/CT has been considered helpful for the management of patients with suspected malignancy. The present study proved both Diam-max and SUVmax of mesenteric nodules were significant independent factors for predicting malignancy in misty mesentery, while there was moderate correlation between the two parameters. These parameters had comparable diagnostic performance when estimated by area under the ROC curve, whereas SUVmax $\geq 3.0$ was a slightly better optimal cut-off point. The combination of 
either Diam-max $>10 \mathrm{~mm}$ or SUVmax $\geq 3.0$ had high sensitivity of $92 \%$ for the identification of viable malignancy. Therefore, misty mesentery with high FDG uptake as well as with large nodules should be targeted for biopsy. Malignancy should be highly suspected if it contains a large nodule with high uptake.

Prior research by Seo et al. using CT data from 29 patients with misty mesentery and the study by Wilkes et al. using CT data from 118 patients with mesenteric panniculitis identified from 73,752 patients indicated the presence of large lymph nodes and the absence of fat ring sign is a predictor of co-existing malignancy $[1,8]$. Our study is distinct in that the purpose was to identify malignant tumor within mesenteric abnormalities themselves, and the majority of patients undergoing PET/CT had malignant tumors that had already been diagnosed. The broader purpose of our study followed that of a CT study by Corwin et al. using data from 37 patients with misty mesentery and that of a descriptive PET/CT study by Zissin et al. using data from 19 oncologic patients with mesenteric panniculitis $[5,6]$, and our statistical results obtained from 71 PET/CT exams were consistent with theirs. Interestingly, the optimal Diammax cut-off value of $>10 \mathrm{~mm}$ calculated by ROC analysis in our study was identical to the cut-off point used in Corwin's study. Small soft tissue nodules scattered within benign mesenteric panniculitis is usually less than $5 \mathrm{~mm}$ [9], and several articles have recommended that nodules over $10 \mathrm{~mm}$ should be biopsied $[7,10]$.

The majority of malignancy within misty mesentery was malignant lymphoma in the present study. Follicular lymphoma generally predominates in the mesentery, where it 
should not be considered as primary gastrointestinal lymphomas [11]. Since FDGPET/CT has advantages at whole-body evaluation and is also a helpful adjunct for the detection of an adequate biopsy site, histological confirmation obtained from alternative sites with less-invasive biopsy can avoid direct mesenteric biopsy. Meanwhile, the present study experienced only one recurrent pancreatic cancer case in which misty mesentery implied invasion or metastasis from gastrointestinal malignancies, though several prior studies had found their frequent co-existence with mesenteric panniculitis $[1,8,9]$. Even when misty mesentery was co-existent with gastric or colorectal cancer, lymph node metastasis of these sites was rarely seen. This is because superior mesenteric nodes and juxta-intestinal mesenteric nodes are not on the direct lymphatic drainage pathway of these cancers. Additionally, peritoneal dissemination without bulky mass did not cause misty change at mesenteric fat in our study. It is presumed that misty mesentery might be a manifestation of abnormality not at peritoneal cavity but at subperitoneal space.

The term misty mesentery is originally a radiological term and considered to be a manifestation mainly of mesenteric panniculitis if neoplastic or other inflammatory causes have been excluded $[2,7]$. Mesenteric panniculitis is a chronic inflammatory condition of unknown etiology and represents an acute form of sclerosing mesenteritis [12]. Imaging mimics of mesenteric panniculitis include primary mesenteric neoplasms such as neurofibroma, lipoma, and mesenteric liposarcoma, which radiologists should carefully distinguish by their visible mass effect on adjacent mesenteric vessels [13]. 
Sclerosing mesenteritis has another two subtypes of histopathological variants within a single spectrum: mesenteric lipodystrophy (fat necrosis dominant) and retractile mesenteritis (fibrosis dominant) $[14,15]$. The present study had excluded mesenteric abnormalities with a bulky mass over $2 \mathrm{~cm}$ in the short-axis diameter, a commonly used cut-off point; however, retractile mesenteritis has extensive fibrosis and sometimes manifests as a large mass. There was a case report that mass-forming sclerosing mesenteritis (probably retractile mesenteritis) mimicking carcinoid tumor had shown FDG uptake [16]. In such a case, it is usually a solitary spiculated mass occasionally seen with calcification associated with fatty necrosis, and unlike lymphoma multiple masses are less common $[3,7,17]$. Other imaging mimics of retractile mesenteritis include aggressive fibromatosis, desmoid tumors, and peritoneal carcinomatosis, the imaging characteristics of which usually differ from misty mesentery [13].

"Fat ring sign" is an alternative radiological finding considered helpful for the assessment of misty mesentery[3], which has been formerly considered pathognomonic for mesenteric panniculitis, but it cannot eliminate the possibility of lymphoma [4]. Mesenteric lymphoma often accompanies retroperitoneal lymphadenopathy, whilst retroperitoneal involvement is uncommon in mesenteric panniculitis [7]. The present study results are inapplicable to retroperitoneal abnormality, but these findings might also be useful for a diagnosis.

Our study has some limitations. As a retrospective PET/CT study, the majority of study population had a history of malignancy and a morbidity of misty mesentery might 
have been different from that of healthy humans. Low dose non-enhanced CT as part of PET/CT was used for image interpretation and so faint opacity of mesenteric fat might have been obscured by image noise in some equivocal cases. Manual assessment on transaxial images was used for measurement of lymph node short-axis diameter instead of semi-automated 3D measurement. PET/CT fusion is not always a perfect method to prevent misregistration by which SUV measurement of small lesions might have been affected. Misty mesentery was categorized into 'viable malignancy' and 'stable lesions' in our study. Stable lesions based on at least 6 months' follow-up would not always be identical to benign lesions, because follicular lymphoma is known as slow-growing lymphoma. Histological confirmation was mainly obtained from alternative lesion sites instead of direct mesenteric biopsy, whereas it is generally accepted as a less-invasive method for the diagnosis of lymphoma. In practice, two or more different subtypes of lymphoma can be present in a single patient. Our data did not exclude the possibility that some rare conditions such as mesenteric lymphatic spread from mucinous cancer (e.g. mucinous ovarian cancer, appendical cancer) might show insignificant uptake of FDG; however, accompanying disseminated nodules at peritoneal fossae would help the diagnoses of malignancy.

\section{Conclusions}

FDG-PET/CT would be feasible for identifying viable malignancy in misty mesentery. If misty mesentery is identified by chance in patients undergoing PET/CT, not only large mesenteric nodules but also the nodules with high FDG uptake are 
recommended to be a target for biopsy. Since malignant lymphoma accounts for the majority of malignancy within misty mesentery, alternative biopsy sites determined by FDG-PET/CT would be permissible.

Conflict of Interest: none 


\section{References}

[1] Seo BK, Ha HK, Kim AY, et al. Segmental misty mesentery: analysis of CT features and primary causes. Radiology 2003; 226(1):86-94.

[2] Mindelzun RE, Jeffrey RB Jr, Lane MJ, Silverman PM. The misty mesentery on CT: differential diagnosis. AJR Am J Roentgenol 1996;167(1):61-5.

[3] Sabaté JM, Torrubia S, Maideu J, Franquet T, Monill JM, Pérez C. Sclerosing mesenteritis: imaging findings in 17 patients. AJR Am J Roentgenol 1999;172(3):625-9.

[4] Valls C. Fat-ring sign in sclerosing mesenteritis. AJR Am J Roentgenol 2000;174(1):259-60.

[5] Corwin MT, Smith AJ, Karam AR, Sheiman RG. Incidentally detected misty mesentery on CT: risk of malignancy correlates with mesenteric lymph node size. J Comput Assist Tomogr 2012;36(1):26-9.

[6] Zissin R, Metser U, Hain D, Even-Sapir E. Mesenteric panniculitis in oncologic patients: PET-CT findings. Br J Radiol 2006;79(937):37-43.

[7] Wat SY, Harish S, Winterbottom A, Choudhary AK, Freeman AH. The CT appearances of sclerosing mesenteritis and associated diseases. Clin Radiol 2006;61(8):652-8.

[8] Wilkes A, Griffin N, Dixon L, Dobbs B, Frizelle FA. Mesenteric panniculitis: a paraneoplastic phenomenon? Dis Colon Rectum 2012;55(7):806-9. 
[9] Daskalogiannaki M, Voloudaki A, Prassopoulos P, et al. CT evaluation of mesenteric panniculitis: prevalence and associated diseases. AJR Am J Roentgenol 2000;174(2):427-31.

[10] van Breda Vriesman AC, Schuttevaer HM, Coerkamp EG, Puylaert JB. Mesenteric panniculitis: US and CT features. Eur Radiol 2004;14(12):2242-8.

[11] van Krieken JH, Otter R, Hermans J, et al. Malignant lymphoma of the gastrointestinal tract and mesentery. A clinico-pathologic study of the significance of histologic classification. NHL Study Group of the Comprehensive Cancer Center West. Am J Pathol 1989;135(2):281-9.

[12] Pickhardt PJ, Bhalla S. Unusual nonneoplastic peritoneal and subperitoneal conditions: CT findings. Radiographics 2005;25(3):719-30.

[13] McLaughlin PD, Filippone A, Maher MM. The "misty mesentery": mesenteric panniculitis and its mimics. AJR Am J Roentgenol. 2013;200(2):W116-23.

[14] Horton KM, Lawler LP, Fishman EK. CT findings in sclerosing mesenteritis (panniculitis): spectrum of disease. Radiographics 2003;23(6):1561-7.

[15] Emory TS, Monihan JM, Carr NJ, Sobin LH. Sclerosing mesenteritis, mesenteric panniculitis and mesenteric lipodystrophy: a single entity? Am J Surg Pathol 1997;21(4):392-8.

[16] Nguyen BD. F-18 FDG PET demonstration of sclerosing mesenteritis. Clin Nucl Med 2003;28(8):670-1. 
[17] Kani KK, Moshiri M, Bhargava P, Kolokythas O. Extrahepatic, nonneoplastic, fat-containing lesions of the abdominopelvic cavity: spectrum of lesions, significance, and typical appearance on multidetector computed tomography. Curr Probl Diagn Radiol 2012;41(2):56-72. 
Fig. 1-68-year-old male with mesenteric panniculitis. Typical axial CT appearance of misty mesentery is shown.

Fig. 2-Flow chart for the selection of PET/CT exams eligible for the present study.

Fig. 3-59-year-old male with 'viable malignancy' in misty mesentery (a: axial CT image, b: axial PET image, c: axial PET/CT fusion image, d: maximum intensity projection PET image). Enlarged mesenteric nodes (Diam-max $=12 \mathrm{~mm}$ ) with increased FDG uptake (SUVmax $=4.5$ ) in the misty mesentery could be identified. Para-aortic node swelling outside mesentery and right hydronephrosis were also observed. He also had right inguinal lymphadenopathy of which he underwent biopsy, and follicular lymphoma grade 1 was confirmed. After 4 cycles of chemotherapy, subsequent CT images showed significant reduction of the mesenteric nodes.

Fig. 4-68-year-old male with 'stable lesions' in misty mesentery (a: axial CT image, b: axial PET image, c: axial PET/CT fusion image, d: maximum intensity projection PET image). Bean-shaped mesenteric nodes (Diam-max $=4 \mathrm{~mm}$ ) with insignificant FDG uptake (SUVmax = 1.3) in the mesentery could be identified. "Fat ring sign" was also observed on the CT image. He underwent direct surgical biopsy of the mesentery, and the histological diagnosis was mesenteric panniculitis. Follow-up CT scans showed no change thereafter. 
Fig. 5-Scatterplots of Diam-max and SUVmax in the 71 patients analyzed with misty mesentery. Black rhombuses represent data in patients with viable malignancy, and white circles represent those with stable lesions. Note those patients with viable malignancy tended to have mesenteric nodules with significantly larger Diam-max and significantly higher SUVmax. The correlation between Diam-max and SUVmax was moderate but significant $(\mathrm{r}=0.69, \mathrm{p}<0.0001)$.

Fig. 6-Receiver-operating characteristic curves of Diam-max and SUVmax for the diagnosis of viable malignancy in misty mesentery. Area under the curves of Diam-max and SUVmax were almost comparable: 0.961 and 0.926, respectively. 
Table 1 Profile of the 71 patients analyzed

\begin{tabular}{|c|c|c|}
\hline \multirow[t]{2}{*}{ Gender } & Male & 47 \\
\hline & Female & 24 \\
\hline \multirow[t]{2}{*}{ Age } & Range $39-88$ & \\
\hline & Mean 65.0 & \\
\hline \multirow[t]{2}{*}{ History of chemotherapy } & Present (not within 3 months) & 31 \\
\hline & Absent & 40 \\
\hline \multirow[t]{13}{*}{ Underlying disease } & Malignant lymphoma & 24 \\
\hline & Malignant melanoma & $2 *$ \\
\hline & Head and neck cancer & 3 \\
\hline & Esophageal cancer & 3 \\
\hline & Breast cancer & 1 \\
\hline & Lung cancer & 7 \\
\hline & Hepatic cancer & 5 \\
\hline & Bile duct /Gallbladder cancer & 5 \\
\hline & Pancreatic cancer & 5 \\
\hline & Colorectal cancer & 6 \\
\hline & Uterine cancer & 2 \\
\hline & Gastrointestinal stromal tumor & 1 \\
\hline & None & 8 \\
\hline
\end{tabular}

*One patient with malignant melanoma had concurrent lymphoma. 
Table 2 Diagnostic ability of PET/CT using each parameter

\begin{tabular}{|c|c|c|c|}
\hline Parameter and cut-off & Sensitivity & Specificity & Accuracy \\
\hline Diam-max $>10 \mathrm{~mm}$ & $69 \% \quad(9 / 13)$ & $98 \%(57 / 58)$ & $93 \%(66 / 71)$ \\
\hline SUVmax $\geq 3.0$ & $85 \%(11 / 13)$ & $98 \%(57 / 58)$ & $96 \%(68 / 71)$ \\
\hline Both* & $62 \% \quad(8 / 13)$ & $100 \%(58 / 58)$ & $93 \%(66 / 71)$ \\
\hline Either $\dagger$ & $92 \%(12 / 13)$ & $97 \%(56 / 58)$ & $96 \%(68 / 71)$ \\
\hline
\end{tabular}

* Both Diam-max $>10 \mathrm{~mm}$ and SUVmax $\geq 3.0$ were required as positive criteria

$\dagger$ Either Diam-max $>10 \mathrm{~mm}$ or SUVmax $\geq 3.0$ was to be sufficient for a positive result 
Table 3 Multivariate logistic regression results among 13 patients with 'viable malignancy' and 58 patients with 'stable lesions'

\begin{tabular}{|c|c|c|c|c|c|c|}
\hline & \multicolumn{5}{|c|}{ Entry of model } & Final model \\
\hline & $\begin{array}{l}\text { Partial } \\
\text { regression } \\
\text { coefficient }\end{array}$ & $\mathrm{P}$ & $\begin{array}{l}\text { Odds ratio } \\
{[95 \% \mathrm{CI}]}\end{array}$ & $\begin{array}{l}\text { Partial } \\
\text { regression } \\
\text { coefficient }\end{array}$ & $\mathrm{P}$ & $\begin{array}{r}\text { Odds ratio } \\
{[95 \% \mathrm{CI}]}\end{array}$ \\
\hline Gender & 0.82 & 0.54 & $\begin{array}{r}2.26 \\
{[0.17,30.0]}\end{array}$ & - & - & - \\
\hline Age & -0.014 & 0.87 & $\begin{array}{r}0.99 \\
{[0.84,1.16]}\end{array}$ & - & - & - \\
\hline $\begin{array}{l}\text { Past history } \\
\text { of lymphoma }\end{array}$ & -0.31 & 0.81 & $\begin{array}{r}0.73 \\
{[0.06,8.97]}\end{array}$ & - & - & - \\
\hline Diam-max & 0.45 & $0.020^{*}$ & $\begin{array}{r}1.56 \\
{[1.07,2.28]}\end{array}$ & 0.43 & $0.017 *$ & $\begin{array}{r}1.53 \\
{[1.08,2.18]}\end{array}$ \\
\hline SUVmax & 1.05 & $0.035^{*}$ & $\begin{array}{r}2.85 \\
{[1.08,7.54]}\end{array}$ & 1.04 & $0.033^{*}$ & $\begin{array}{r}2.82 \\
{[1.09,7.30]}\end{array}$ \\
\hline
\end{tabular}

Nagelkerke's $\mathrm{R}^{2}$ at entry of the model and at the final model was 0.79 and 0.78 , respectively.

*, statistically significant; CI, confidence interval 


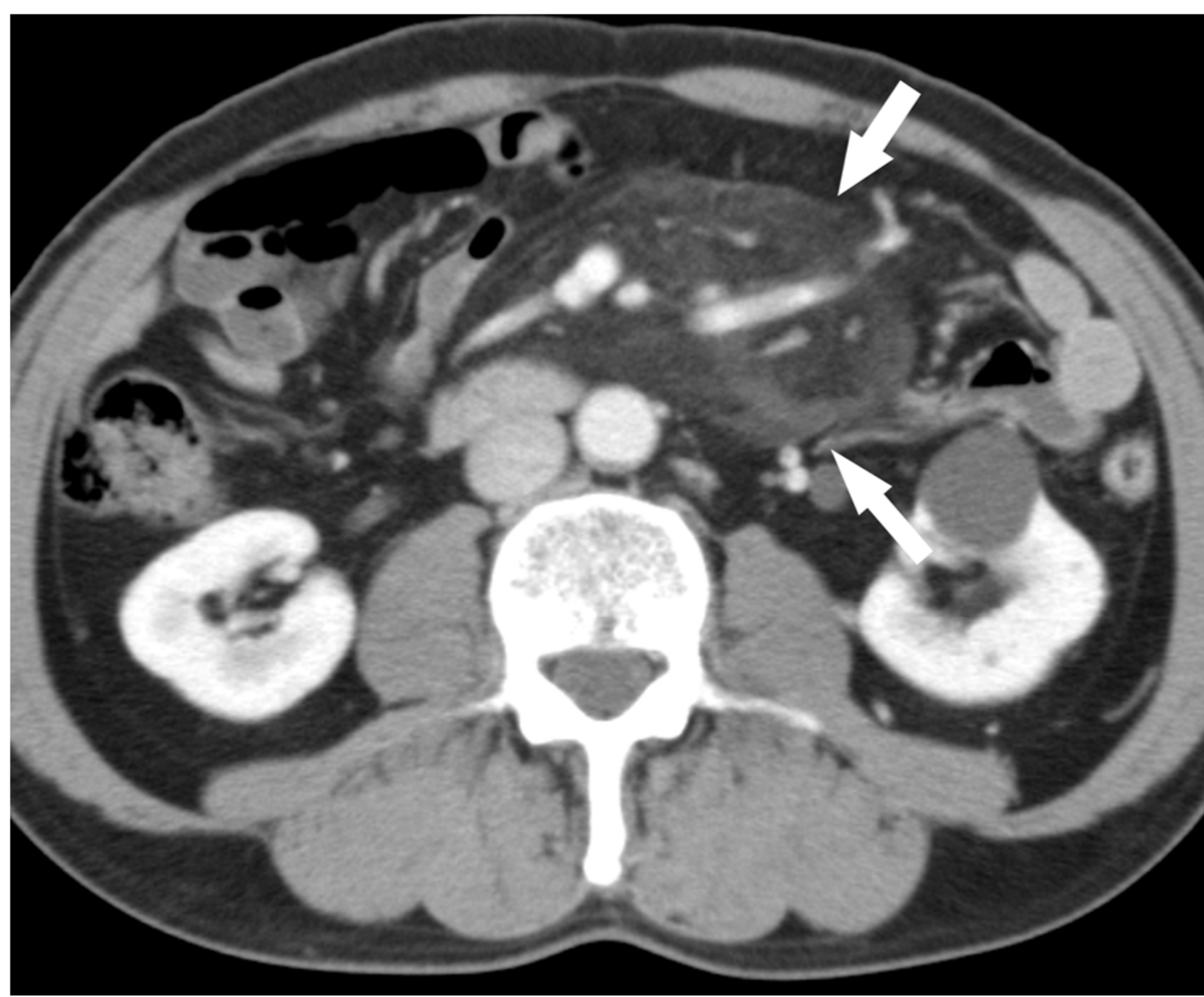

Fig. 1 
Total number of whole-body PET/CT exams

4236 scans

Exams with a CT appearance of a misty mesentery 209 scans

The initial exams with a misty mesentery

153 scans

$<$ Study exclusion criteria>

(a) With a history of chemo- or radiotherapy within 3 mo. 40

(b) With a bulky mesenteric mass

7

(c) With acute abdomen

0

(d) Lack of follow-up CT or PET/CT examinations

Exams eligible for the present study

\section{3 scans}

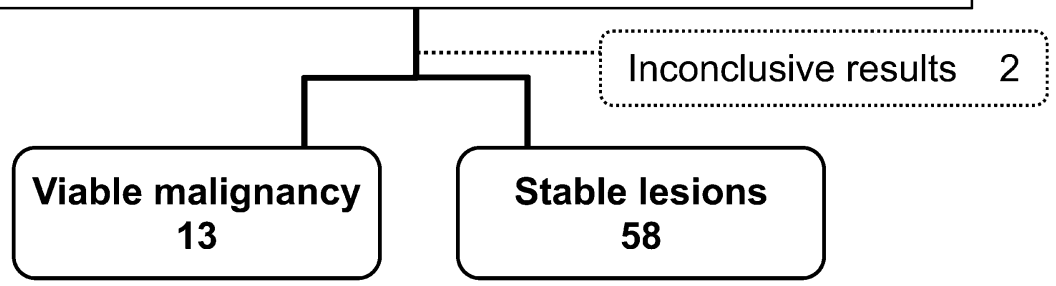




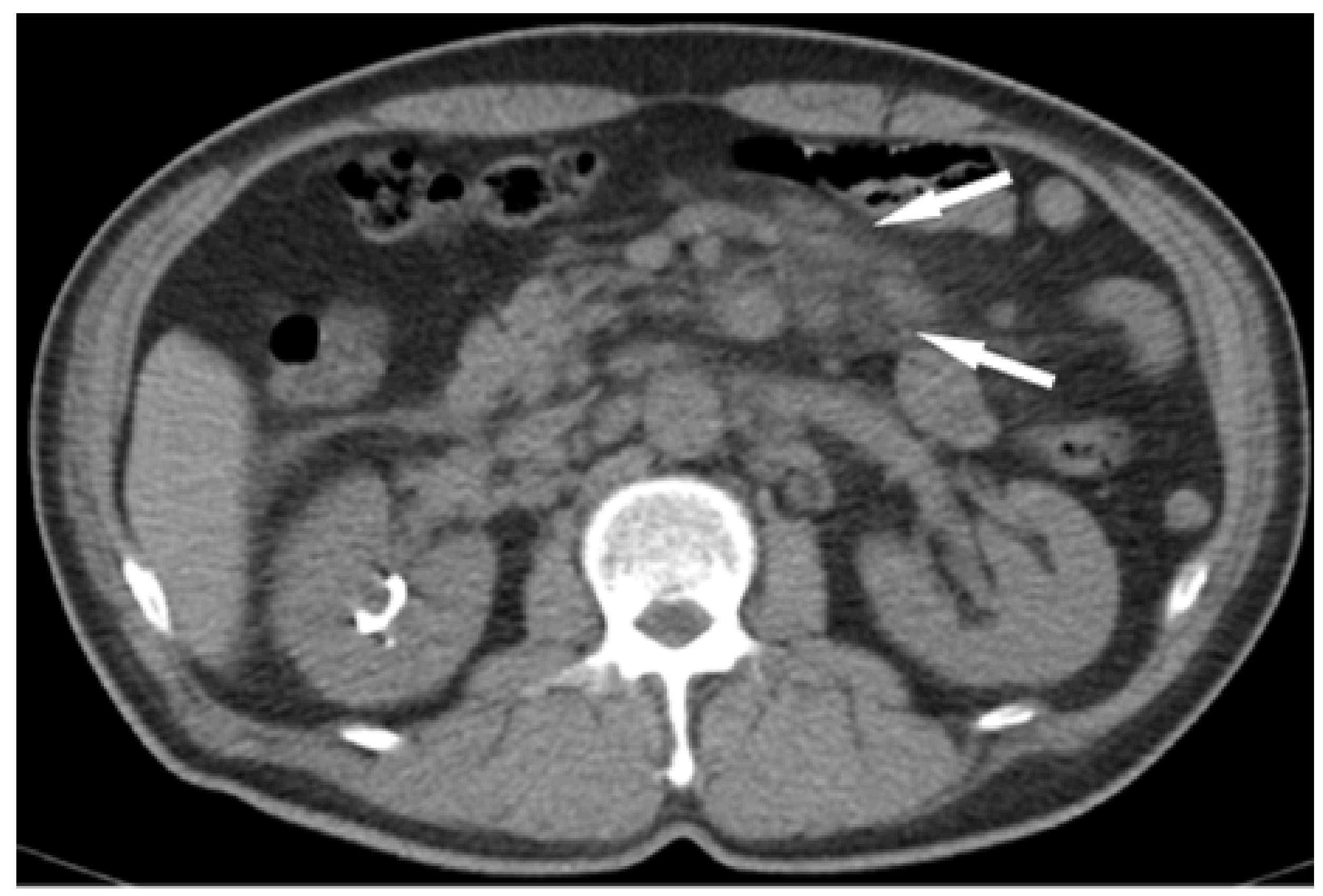

Fig. 3_a 
Fig. 3_b 


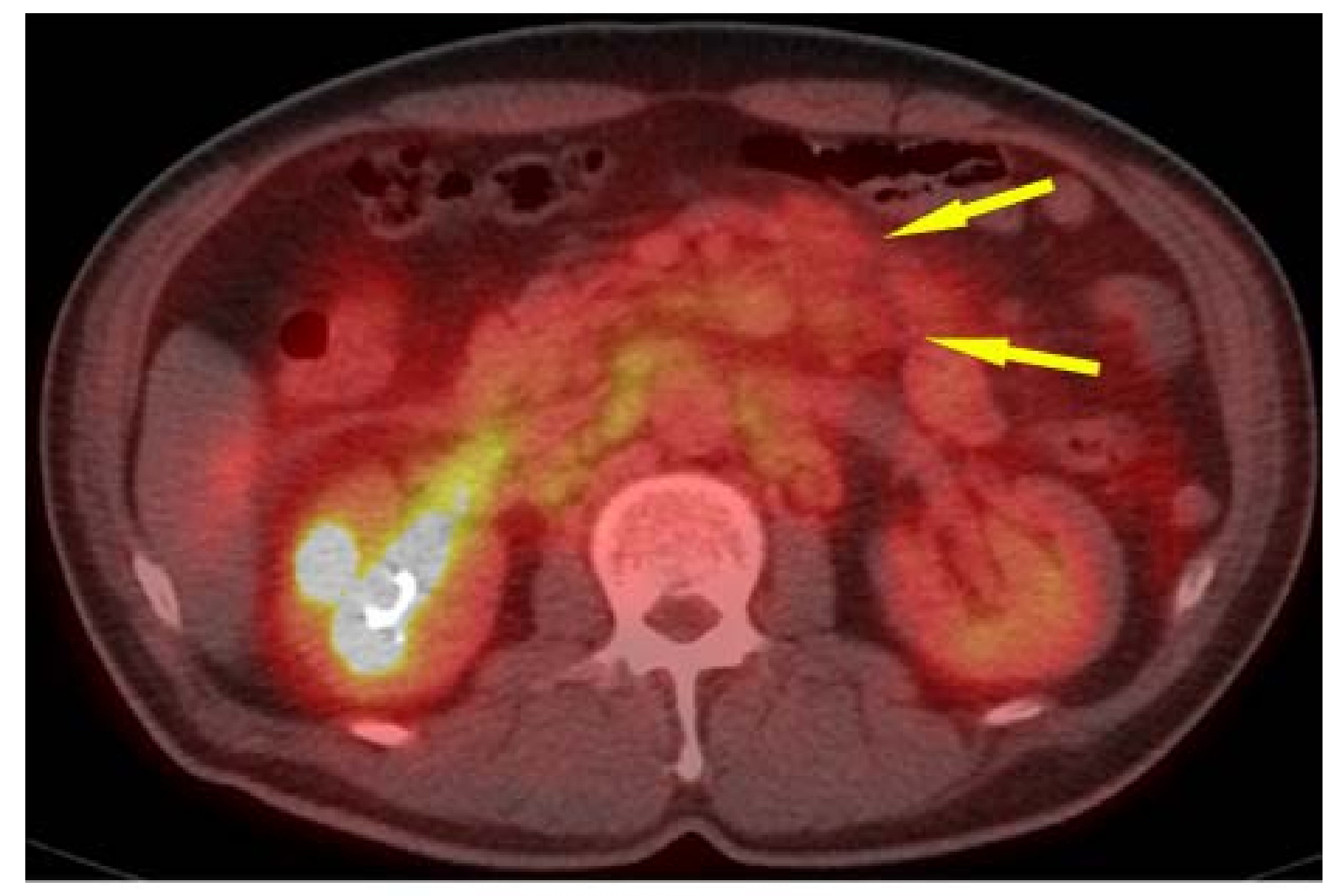

Fig. 3_c 


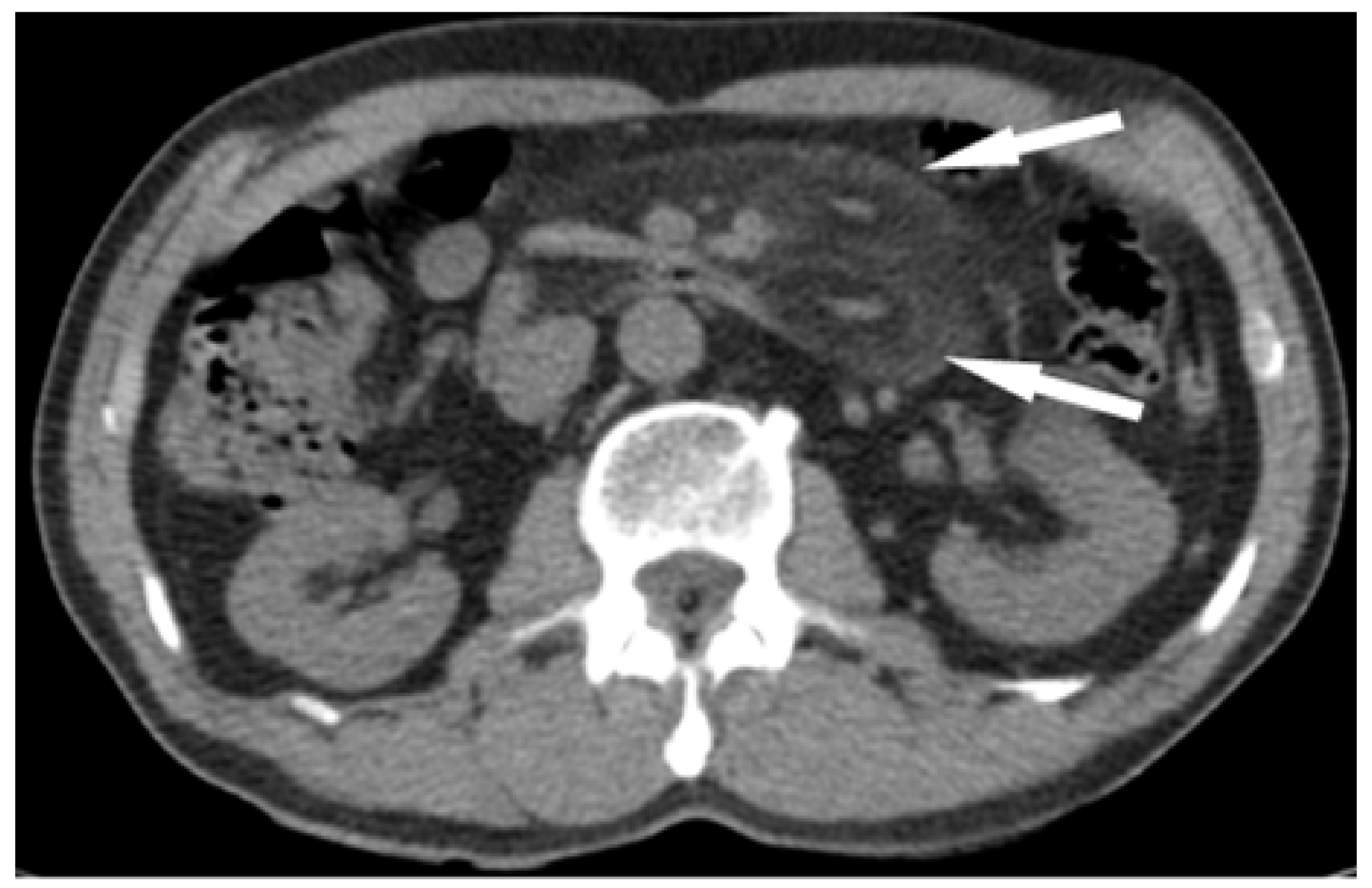

Fig. 4_a 
Fig. 4_b 


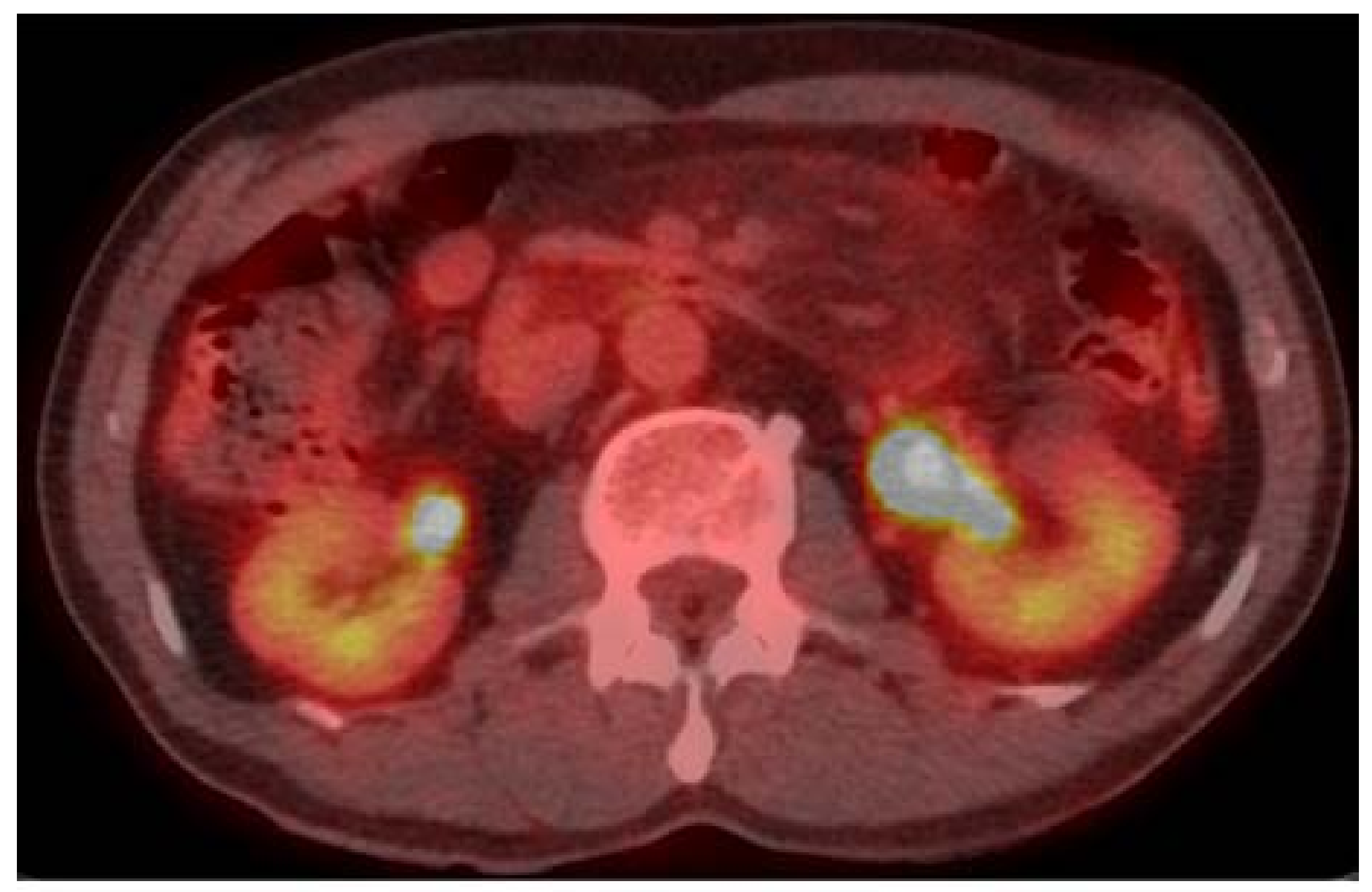

Fig. 4_c 
Fig. 4_d 


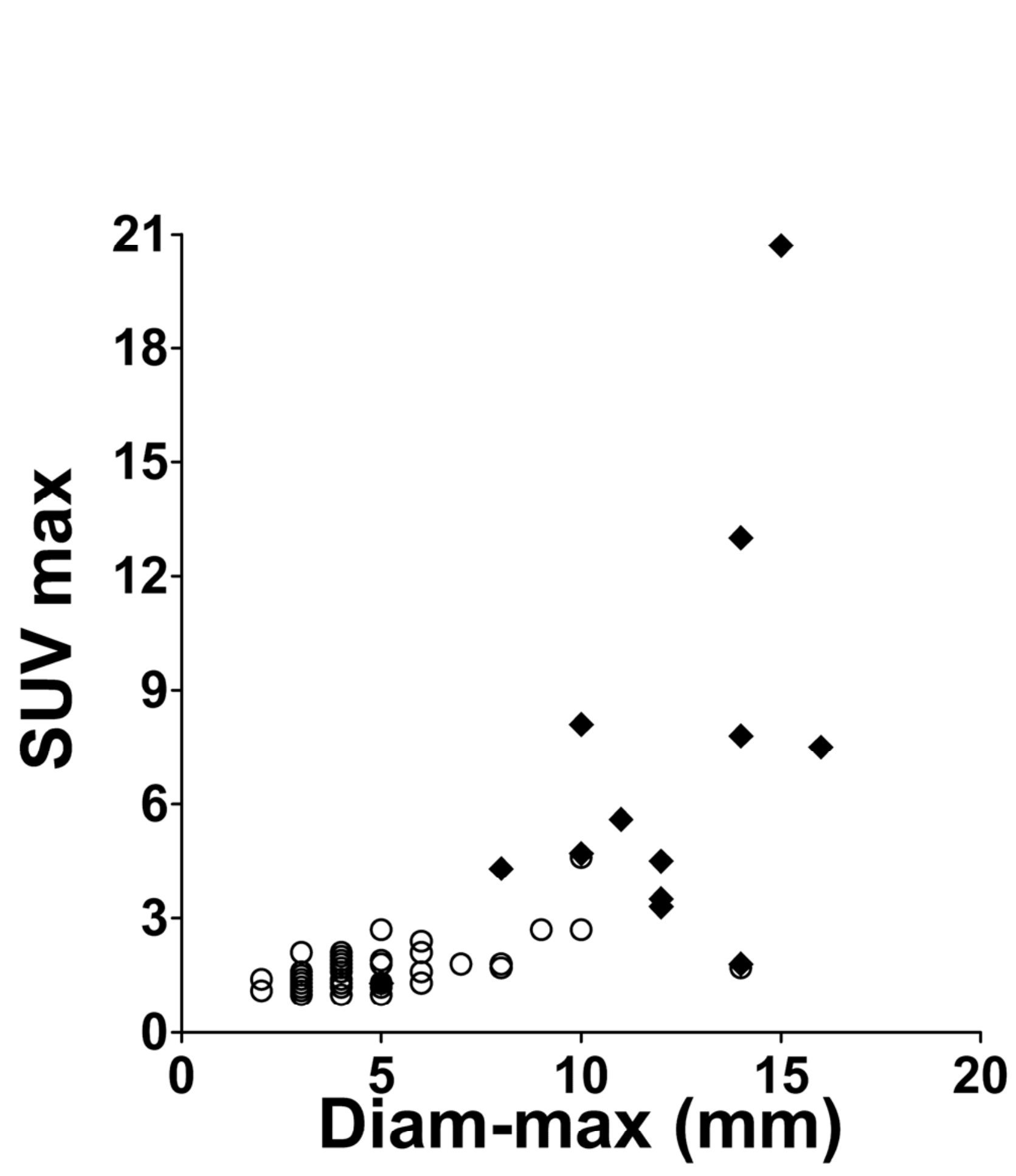

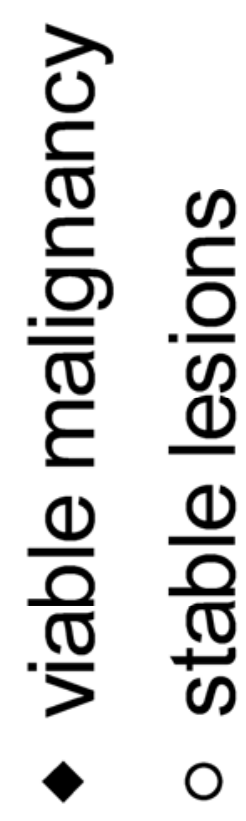

Fig. 5 


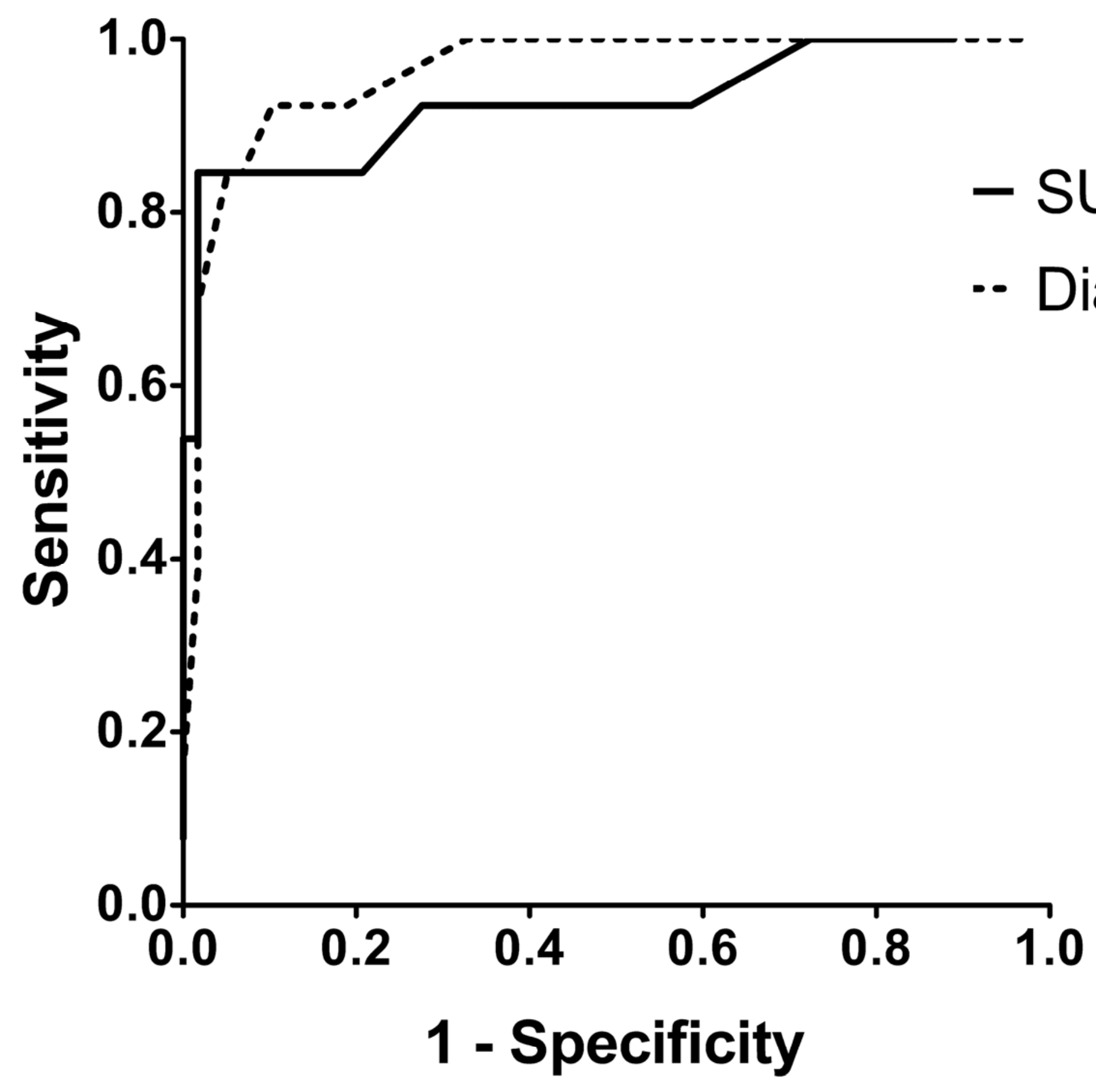

Fig. 6 